\title{
A Novel Technique of Repositioning Papilla to Create a Balanced Anterior Pink and White Esthetics
}

\author{
Shrestha $\mathrm{P}^{1^{*}}$, Shakya $\mathrm{S}^{2}$ \\ ${ }^{1}$ Associate Professor, Department of Prosthodontics and Maxillofacial Prosthetics \\ ${ }^{2}$ Assistant Professor, Department of Periodontics and Oral Implantology \\ KIST Medical College and Hospital, Lalitpur, Nepal.
}

\begin{abstract}
The ultimate goal in modern era dentistry is to achieve the balance of "white" and "pink" in esthetically important zones. "White esthetics" is the natural dentition or the restoration of dental hard tissues with suitable materials. "Pink esthetics" refers to the surrounding soft-tissues, which includes the interdental papilla and gingiva that can enhance or diminish the esthetic result. This paper aims to describe a careful diagnosis and multidisciplinary treatment approach to achieve a satisfactory final esthetic outcome in a clinical scenario where there is an abnormal shape, position of tooth and displaced papilla in the anterior esthetic zone.
\end{abstract}

Key words: Papilla, Pink and white esthetics, Teflon tape, Veneer.

\section{Introduction}

Qver the past several years, esthetic awareness has increased greatly and dentistry has developed various techniques to fulfill the patient's esthetic demands. The modern era dentistry has been converted to pink and white esthetic dentistry. The use of ceramic veneers has proven to be an effective treatment option to manage the esthetic and functional problems with respect to white esthetics, especially in the anterior region ${ }^{1}$. Moreover, its use has increased due to color and contour stability, superior esthetics, and the involvement of minimal tooth preparation ${ }^{2}$. Color, symmetry and size of papilla plays a crucial role in the pink esthetic outcome ${ }^{3,4}$ and gingival levels have direct impact on pink esthetics. There is no doubt that establishing the correct tone for

\section{*Corresponding Author}

Dr. Prabhat Shrestha, Associate Professor Department of Prosthodontics and Maxillofacial Prosthetics, KIST Medical College and Hospital, Lalitpur, Nepal.

E-mail: prabhatshrestha@gmail.com each individual tooth is the key in the creation of harmonious smile 5 .

Various techniques have been developed for the modification / alteration of papilla including nonsurgical and surgical techniques. Among nonsurgical techniques there are orthodontic and restorative techniques ${ }^{6,7}$. In our clinical scenario we may come across cases where only one option might not suffice to bring about the desired outcome. Multiple disciplines including Prosthodontics, Periodontology and dental technology have to be involved in accomplishing the treatment goals. ${ }^{5,7,8}$

The case presented here was done by systematic and multidisciplinary approach. A combination of surgical and restorative techniques was used to modify the position of the papilla and gingival contours, to achieve an esthetic balance. Final restoration was done with minimal preparation lithium disilicate-reinforced glass ceramic veneers (IPS e.max Press, Ivoclar Vivadent, Liechtenstein) to modify the esthetic counters of the teeth. 


\section{Methods (case report)}

A nineteen years old female patient presented with a retained right deciduous maxillary central incisor root stump. The patient was very scared of dental treatment and had been avoiding extraction for a long time. After much counseling, she reluctantly agreed to extract the teeth under local anesthesia.

The patient again came back after a week with confidence from the previous painless experience to correct her smile. On examination she had a large maxillary mid line diastema. The shape size and position of the central incisors were unsymmetrical. The gingival contour of central incisors was uneven with the left being lower than that of right central incisors. She had bilateral missing maxillary lateral incisors and retained deciduous canines. The permanent canines had erupted in place of the lateral incisors (Fig. 1).

The interdental papilla of the central incisors had been shifted towards the left side by the root stump of previous deciduous central incisor (Fig. 2).

Various treatment options were given including orthodontic treatment, extraction of deciduous canines and placement of bridges or implants but she rejected these time consuming and tedious options. She finally decided to only fix the midline diastema with e-max veneers.

The treatment plan was divided into two stages

1. Periodontic intervention: which included repositioning of the interdental papilla towards the mid line and recontouring the gingival margin of central incisors

2. Prosthetic rehabilitation: teeth preparation and veneer placement

The procedures were explained to the patient and diagnostic wax up was done on a study cast. Treatment started after approval of this wax up by the patient.
Shade selection of the teeth was done. A Teflon tape was inserted between the papilla and left central incisors to retract the papilla towards the midline to the desired position (Fig. 3).

Etching of proximal tooth surface was done with 37\% phosphoric acid (Meta Etchant, META BIOMED, korea) for 20 secs. And bonding agent (MetaPand Bond, META BIOMED, UK) was applied. Composite resin (3M ESPE Filtek ${ }^{\mathrm{TM}}$ Z250 universal restorative, 2510 Conway Ave. ST. Paul, MN 55144-1000, USA) was used with free hand to build up the proximal wall for support of the papilla (Fig. 4).

The follow up was done after 2 weeks. Scalpel gingivectomy was performed on left central incisor under local anesthesia to match the gingival zenith of the two central incisors. Minimal preparation of the incisors was done to remove any undercuts without removing the composite restoration. Alginate impression was taken of the wax mockup to make a provisional veneer by direct technique using bis-acryl composite (Cool Temp coltene, UK) by direct method. The incisors were spot etched with $37 \%$ phosphoric acid for 20 secs and the temporaries were luted with bonding agent.

After the gingival healing period which is approximately 5 weeks, the temporaries were removed. Under local anesthesia, final tooth preparation of incisors was done removing all the remaining composite resin (Fig. 5).

A double retraction cord technique (using no.0 Roeko Stay-put non impregnated cord and no. 2 AtriaPak non medicated retraction cord) was performed (Fig. 6).

Final impression was taken using two step impression technique without spacer with vinyl polysiloxane impression material (Perfit Elastomeric impression material Type 0 and Type 3). After the first step the putty in the papilla area was removed and channels were made with bp blade no. 15 for the flow of light 
body to make a mucostatic impression in the papillary region.

The temporary veneers were cemented with flowable composite resin (Beautiful Flow, Sofu Inc., Japan) without using a bonding agent (Fig. 7).

After the lithium disilicate-reinforced glass ceramic veneers (IPS e.max Press, Ivoclar

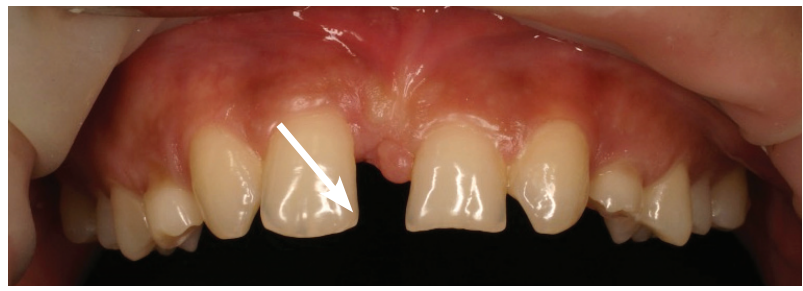

Figure 1: Arrow showing healing site of extracted maxillary deciduous central incisors which caused shifting of the papilla away from the mid line.

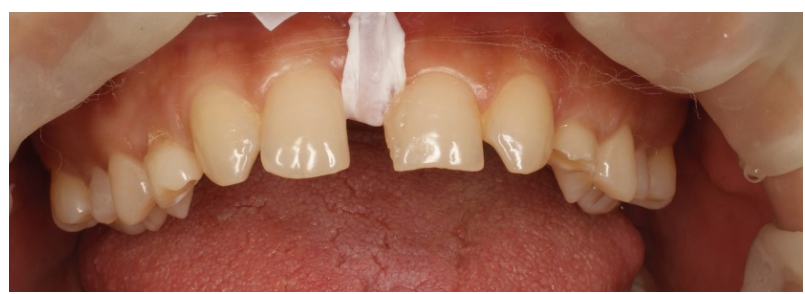

Figure 3: Shifting of the interdental papilla into its original position with Teflon tape and composite build up for its support

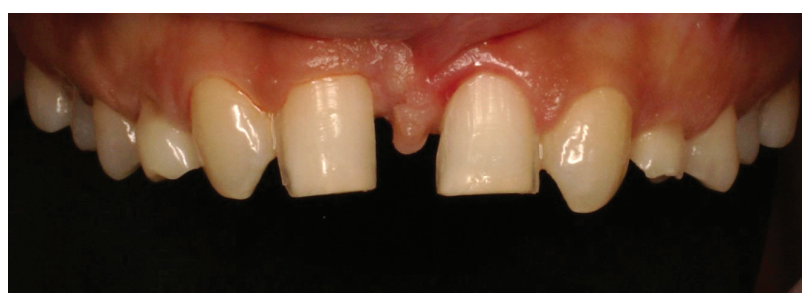

Figure 5: Tooth after preparation for Veneer. Notice that the interdental papilla is stable without any support.

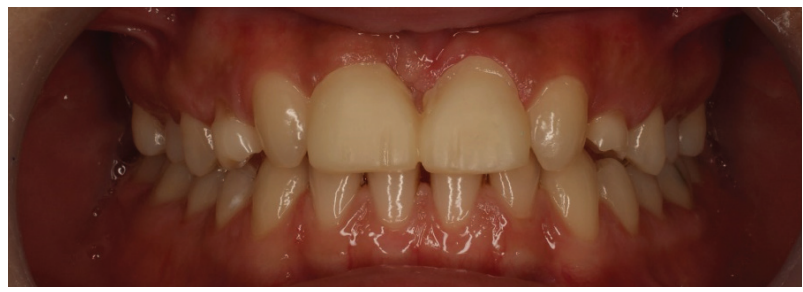

Figure 7: Temporization of prepared central incisors
Vivadent, Liechtenstein), were fabricated from the lab they were trailed and then etched with 9\% hydrofluoric acid (Ultradent porcelain etch, ultradent products Inc, USA) for 10 secs and rinsed, then again etched with 37\% phosphoric acid and rinsed with normal saline. Company protocols were followed to lute the veneers with resin cement (Multilink N, Ivoclar Vivadent, Liechtenstein) (Fig. 8).

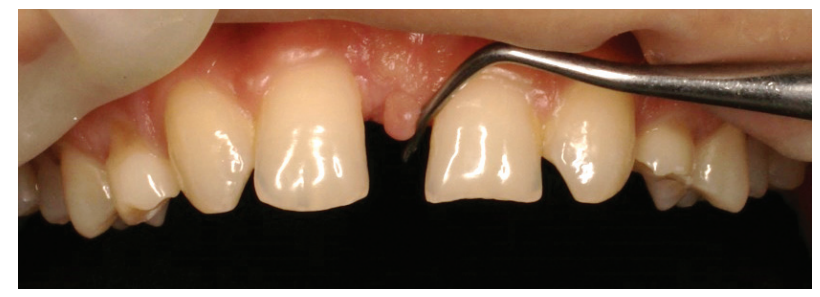

Figure 2: Shifting the interdental papilla into its actual position with a probe

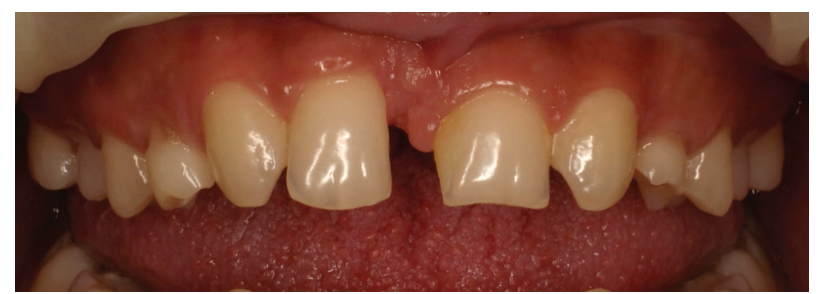

Figure 4: Composite resin stabilizing the papilla in its desired position

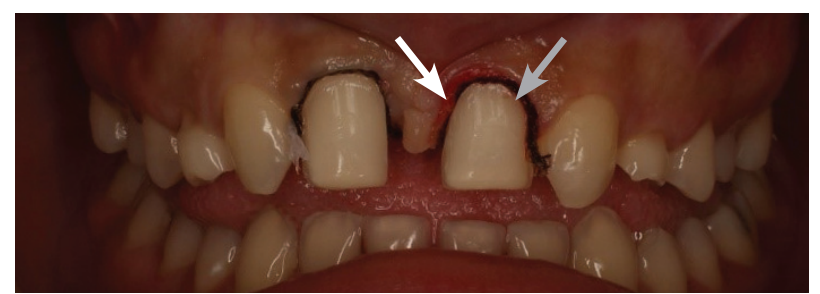

Figure 6: White arrow shows no. 0 gingival retraction cord and Gray arrow showing no. 2 gingival retraction cord

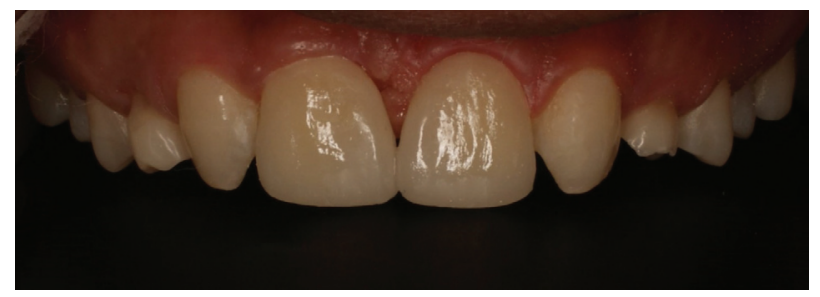

Figure 8: Final outcome after cementation of veneer Journal of Nepalese Prosthodontic Society (JNPS) 


\section{Discussion}

The above presented case was an uncommon one in which the papilla had been displaced from its original position by the root of deciduous central incisor. This simple technique used Teflon tape and Composite resin to transfer and maintain the position of the papilla to a more desirable position for esthetic improvement.A nice emergence profile could be maintained by this restorative approach.

The use of Teflon tape to retract and keep the papilla in desired place is an innovative technique which also helps to protect the soft tissues during etching with acid and prevent moisture contamination. The Teflon tape overcomes the impracticality of using rubber dam as a means of isolation during the repositioning of papilla.

Proper design of final restoration is also crucial for the long term success of treatment outcome. Circular (oval) teeth were designed in this case, which produced a shallower gingival scallop rather than triangular teeth which forms a pronounced scallop and predisposes to the so-called 'black triangles'. This is similar to an Ovate pontic which serves in maintaining the interdental papilla next to abutment teeth after extraction. The ovate pontic helps to create or maintain the interdental papilla. It is also effective design for cleansibility and also eliminates 'black triangle' spaces. ${ }^{10}$

\section{Conclusion}

Esthetically complex cases especially in the anterior region require a multidisciplinary approach.This article demonstrates a protocol of a novel approach for repositioning the papilla and maintaining its position throughout the various phases of clinical execution to maintain a balanced pink and white esthetic outcome.

\section{References}

1. Kamble V, Parkhedkar R. Esthetic rehabilitation of discolored anterior teeth with porcelain veneers. Contemp Clin Dent [Internet]. 2013;4(1):124. Available from: http://www.contempclindent.org/text. asp?2013/4/1/124/111635

2. Rotoli B, Lima D, Pini N, Aguiar F, Pereira G, Paulillo L. Porcelain Veneers as an Alternative for Esthetic Treatment: Clinical Report. Oper Dent [Internet]. 2013 Sep;38(5):459-66. Available from: http://www.jopdentonline.org/ doi/10.2341/12-382-T

3. Yu YCP, Alamri A, Francisco H, Cho S, Hirsch S. Interdental Papilla Length and the Perception of Aesthetics in Asymmetric Situations. Int J Dent [Internet]. 2015;2015:1-5. Available from: http://www.hindawi.com/journals/ ijd/2015/125146/

4. Frank Spear, DDS M. Embrasure and Papilla Form in Anterior Esthetics [Internet]. speareducation.com. 2017. p. 6. Available from: https://kinzerrousespear.com/pdf/materialsand-techniques/Spear-Embrasure_\&_Papilla Form_in_Anterior_Esthetics.pdf

5. Daouahi N, Hadyaoui D. Achieving Harmony between Pink and White in Fixed Prosthodontics. SM J Dent. 2015;1(1):1-2.

6. Singh VP, Uppoor AS, Nayak DG, Shah D. Black triangle dilemma and its management in esthetic dentistry. Dent Res J (Isfahan) [Internet]. 2013;10(3):296-301. Available from: http://www.pubmedcentral.nih.gov/ articlerender.fcgi?artid $=3760350 \&$ tool $=$ pmcen trez\&rendertype $=$ abstract

7. Sharma AA, Park JH. Esthetic Considerations in Interdental Papilla: Remediation and Regeneration. J Esthet Restor Dent [Internet]. 2010 Feb;22(1):18-28. Available from: http://doi.wiley.com/10.1111/j.17088240.2009.00307.x

8. Lee EA, Jun SK. Achieving aesthetic excellence through an outcome-based restorative treatment rationale. Pract Periodontics Aesthet Dent. 2000;12(7):641-8; quiz 650.

9. Ahmad I. Anterior dental aesthetics: Gingival perspective. Br Dent J. 2005;199(4):195-202.

10. Banerjee R, Banerjee S, Radke U. Ovate Pontic Design: An Aesthetic Solution to Anterior Missing Tooth-A Case Report. Vol. 4, Journal of Clinical and Diagnostic Research. 2010. 2996-2999 p. 